Mathematical Research Letters 7, 709-717 (2000)

\title{
LINE BUNDLES, RATIONAL POINTS AND IDEAL CLASSES
}

\author{
A. Agboola and G. Pappas
}

In this note, we will use the term "arithmetic variety" for a normal scheme $X$ for which the structure morphism $f: X \rightarrow \operatorname{Spec}(\mathbb{Z})$ is proper and flat. Let $V$ be a proper, normal (not necessarily geometrically connected) variety over $\mathbb{Q}$. Let us choose a normal model for $V$ over $\mathbb{Z}$, that is an arithmetic variety $X$ whose generic fiber is identified with $V$. Suppose that $F$ is a number field and consider the $F$-rational points of $V$. These correspond bijectively to $R$-valued points of $X$, with $R$ the ring of integers of $F$. If $P$ is an $F$-rational point of $V$, we will also denote by $P: \operatorname{Spec}(R) \rightarrow X$ the corresponding $R$-valued point of $X$.

Suppose that $\mathcal{L}$ is a line bundle on the arithmetic variety $X$. We say that $\mathcal{L}$ is trivial, when it is isomorphic to the structure sheaf $\mathcal{O}_{X}$. We will denote by $P^{*} \mathcal{L}$ the pull-back of $\mathcal{L}$ to $\operatorname{Spec}(R)$ via the morphism $P$; then $P^{*} \mathcal{L}$ is a line bundle on $\operatorname{Spec}(R)$. It gives an element $\left(P^{*} \mathcal{L}\right)$ in the class group $\operatorname{Pic}(R)$ of $R$. In what follows, we will identify $\operatorname{Pic}(R)$ with the ideal class group $\mathrm{Cl}(F)$. This paper is motivated by the following question of the second named author:

Question. Suppose that the line bundle $\mathcal{L}$ on $X$ is not trivial. Is there a number field $F$ and an $F$-rational point $P$ of $V$ such that the ideal class $\left(P^{*} \mathcal{L}\right)$ is not trivial?

As a variant of this question, we could also ask: Is there a scheme $Z$ which is finite and flat over $\operatorname{Spec}(\mathbb{Z})$ and a morphism $P: Z \rightarrow X$ such that $\left(P^{*} \mathcal{L}\right)$ is not trivial in $\operatorname{Pic}(Z)$ ?

L. Szpiro has informed us that he independently raised this question earlier. If the answer to the question is always positive, then line bundles on arithmetic varieties are characterized by their restrictions to integral points. Here are some interesting facts about this question:

1. The answer is positive when $X=\mathbb{P}^{n}$. Indeed, restricting along any linear morphism $\mathbb{P}^{1} \rightarrow \mathbb{P}^{n}$ gives an isomorphism on Picard groups. Therefore, it is enough to show the statement for $X=\mathbb{P}^{1}$. Take $\mathcal{L}=\mathcal{O}_{X}(n), n \neq 0$. There is a number field $F$ with an ideal $\mathcal{A}$ whose ideal class is not $n$-torsion. We can always write $\mathcal{A}=a \mathcal{O}_{F}+b \mathcal{O}_{F}$ with $a, b$ in $\mathcal{O}_{F}$. Consider the $F$-rational point $(a ; b)$ of $\mathbb{P}^{1}$. This gives a morphism

$$
P: \operatorname{Spec}\left(\mathcal{O}_{F}\right) \rightarrow \mathbb{P}_{\mathcal{O}_{F}}^{1} \rightarrow \mathbb{P}_{\mathbb{Z}}^{1}
$$

Received May 17, 2000. 
Then the pull-back of $\mathcal{L}=\mathcal{O}_{X}(n)=\mathcal{O}_{X}(1)^{\otimes n}$ is the line bundle on $\operatorname{Spec}\left(\mathcal{O}_{F}\right)$ which corresponds to $\mathcal{A}^{n}$; this is not trivial.

Now suppose that $X$ is a subscheme of projective space $\mathbb{P}^{n}$ defined by the homogeneous polynomials $f_{1}, \ldots, f_{k}$ in $\mathbb{Z}\left[x_{0}, \ldots, x_{n}\right]$. Let $\mathcal{L}=\mathcal{O}_{X}(m)=$ $\mathcal{O}_{P^{n}}(m) \mid X$. The question then becomes:

Is there a number field $F$ and an ideal $\mathcal{A} \subset \mathcal{O}_{F}$ generated by elements $a_{0}, \ldots, a_{n}$ such that:

A) the ideal class $(\mathcal{A})$ is not $m$-torsion, and

B) $\left(a_{0}, \ldots, a_{n}\right)$ is a zero of the polynomials $f_{1}, \ldots, f_{k}$ ?

We observe that a positive answer to our question is closely related to exhibiting ideal classes in number fields which are generated by elements which satisfy a given (a priori) set of polynomial equations.

2. Let $N$ be a natural number which is coprime to 6 . The results in $[\mathrm{A}]$ and $[\mathrm{P}]$ show that if $X=\mathcal{E}$ is an elliptic curve over the ring of integers $\mathcal{O}_{K}$ of a number field, then any $N$-torsion line bundle on $\mathcal{E}$ whose restriction to the identity section is trivial restricts to the trivial line bundle on all torsion points (in fact, for all $m$, it restricts to the trivial line bundle on the finite and flat subscheme of torsion points $\mathcal{E}[m])$.

3. Suppose that $f$ has a Stein factorization $X \stackrel{g}{\rightarrow} B \rightarrow \operatorname{Spec}(\mathbb{Z})$. Here $B=\operatorname{Spec}\left(\mathcal{O}_{K}\right)$, where $\mathcal{O}_{K}$ is the ring of integers of a number field $K$. Let $\mathcal{A}$ be an ideal in $\mathcal{O}_{K}$ and take $\mathcal{L}$ to be the pull-back $g^{*} \widetilde{\mathcal{A}}$ of the corresponding line bundle $\widetilde{\mathcal{A}}$ on $B$. In this situation, since $g^{*}: \operatorname{Pic}(B) \rightarrow \operatorname{Pic}(X)$ is injective, we see that the question above is equivalent to the following question:

Let $V$ be a geometrically connected normal proper variety defined over the number field $K$ and let $\mathcal{A}$ be an non-principal ideal of $K$. Is there a number field $L / K$ with $V(L) \neq \emptyset$ such that $\mathcal{A O}_{L}$ is also not principal?

If there does exist a geometrically connected variety $V$ over $K$ for which the non-principal ideal $\mathcal{A}$ of $K$ becomes principal ("capitulates") in all extensions $L / K$ in which $V$ acquires a rational point, it would make sense to call $V$ a "capitulating variety" for $\mathcal{A}$.

In this note, we show that an "Arakelov variant" of the above question in which we endow the line bundles with hermitian metrics has a positive answer. This Arakelov variant of the question has also been suggested by L. Szpiro. Heuristically speaking, one could view this result as showing that if a line bundle on $X$ is trivialized on integral points "using sections of uniformly bounded length" then the line bundle is trivial.

Denote by $L$ the restriction of $\mathcal{L}$ to the generic fiber $V$. Let $K$ be the (number) field of constants of $V$. For every embedding $\sigma: K \subset \mathbb{C}$ the complex variety $V_{\sigma}:=V \otimes_{K, \sigma} \mathbb{C}$ is a proper connected variety over $\mathbb{C}$ and we have:

$$
X(\mathbb{C})=V(\mathbb{C})=\bigsqcup_{\sigma: K \rightarrow \mathbb{C}} V_{\sigma}(\mathbb{C})
$$


A hermitian line bundle $\overline{\mathcal{L}}$ on $X$ is by definition a pair $\left(\mathcal{L},\|\|_{\mathcal{L}}\right)$ consisting of a line bundle $\mathcal{L}$ on $X$ and a collection \|\|$_{\mathcal{L}}=\left(\|\|_{\sigma}\right)_{\sigma: K \subset \mathbb{C}}$ of continuous hermitian metrics \|\|$_{\sigma}$ on the complex line bundles $L_{\sigma}$ over $V_{\sigma}(\mathbb{C})$ which are given by $L \otimes_{K, \sigma} \mathbb{C}$. We require that this family be invariant under complex conjugation. The isomorphism classes of hermitian line bundles on $X$ form a group (the group operation is given by tensor product) which we denote $\widehat{\operatorname{Pic}}(X)$. The identity is the isomorphism class of the "trivial hermitian line bundle"; this is the structure sheaf $\mathcal{O}_{X}$ with the trivial hermitian metric (i.e. $\|1\|_{\sigma}=1$, for all $\sigma$ ). If $R$ is the ring of integers of a number field, we will call the group $\widehat{\operatorname{Pic}}(\operatorname{Spec}(R))$ the Arakelov class group of $R$.

Theorem 1. Suppose that $\overline{\mathcal{L}}$ is a non-trivial hermitian line bundle on the arithmetic variety $X$. Then, there is a number field $F$ with ring of integers $R$ and a point $P: \operatorname{Spec}(R) \rightarrow X$ such that the pull-back hermitian line bundle $P^{*} \overline{\mathcal{L}}$ is not trivial in the Arakelov class group of $R$.

The main ingredients of the proof are standard results on height functions, a theorem of Rumely on integral points of arithmetic varieties ([Ru]) (see also $[\mathrm{Mo}])$ and the Hilbert irreducibility theorem. Here are some remarks before we give the proof:

1. Recall that there is a natural surjective homomorphism $\phi: \widehat{\operatorname{Pic}}(X) \rightarrow$ $\operatorname{Pic}(X)$ given by forgetting the metrics. One can ask the following question. Given an arithmetic variety $X$, is there a subgroup $\widehat{\operatorname{Pic}}^{\prime}(X) \subset \widehat{\operatorname{Pic}}(X)$ with $\phi\left(\widehat{\operatorname{Pic}}^{\prime}(X)\right)=\operatorname{Pic}(X)$ and a point $P \in X(R)$ for some integer ring $R$ such that the restriction $P^{*}: \widehat{\operatorname{Pic}}^{\prime}(X) \rightarrow \widehat{\operatorname{Pic}}(R)$ is injective? If $X$ is an arithmetic surface with smooth generic fiber a natural candidate for the group $\widehat{\operatorname{Pic}}^{\prime}(X)$ would be the group of hermitian line bundles with admissible (in the sense of Arakelov) metrics.

2. In [Bo] (see Theorem 1.2), J. Bost shows that regular $X$ and for suitably "very positive" points $P: \operatorname{Spec}(R) \rightarrow X$ we obtain a surjection on fundamental groups:

$$
P_{*}: \pi_{1}(\operatorname{Spec}(R), \eta) \rightarrow \pi_{1}(X, \eta)
$$

where $\eta$ is a geometric point of $\operatorname{Spec}(R)$. This can be viewed as a "Lefschetz-type theorem for $\pi_{1}$ "; here we are dealing with Lefschetz-type statements about Pic and $\widehat{\mathrm{Pic}}$.

Recall our notation $X \stackrel{g}{\rightarrow} B \rightarrow \operatorname{Spec}(\mathbb{Z})$ with $B=\operatorname{Spec}\left(\mathcal{O}_{K}\right)$ for the Stein factorization of $f: X \rightarrow \operatorname{Spec}(\mathbb{Z})$. We may define a height function (see [Si], p. 165)

$$
h_{\overline{\mathcal{L}}}: V(\overline{\mathbb{Q}}) \rightarrow \mathbb{R}
$$

in the following way: Suppose that $P \in V(F)$, where $F / K$ is some finite extension. We set $h_{\overline{\mathcal{L}}}(P)=[F: \mathbb{Q}]^{-1} \widehat{\operatorname{deg}}\left(P^{*} \overline{\mathcal{L}}\right)$, where $\widehat{\operatorname{deg}}$ denotes the Arakelov 
degree and where we also denote by $P: \operatorname{Spec}\left(\mathcal{O}_{F}\right) \rightarrow X$ the corresponding $\mathcal{O}_{F^{-}}$ valued point. Up to the addition of a bounded function on $V(\overline{\mathbb{Q}}), h_{\overline{\mathcal{L}}}$ depends only on the class of $L$ in $\operatorname{Pic}(V)$. In order to prove Theorem 1, we will need the following result which is itself of some independent interest:

Theorem 2. The height function $h_{\overline{\mathcal{L}}}: V(\overline{\mathbb{Q}}) \rightarrow \mathbb{R}$ is constant (resp. zero) if and only if for some $m>0, \overline{\mathcal{L}}^{\otimes m}$ is isomorphic to the pull-back by $g$ of a hermitian line bundle (resp. of Arakelov degree 0) on $B$.

Proof of Theorem 2. Since the case in which $\operatorname{dim}(X)=1$ is trivial, we assume that $\operatorname{dim}(X)>1$.

Suppose first that the $m$-th power of $\overline{\mathcal{L}}$ is isomorphic to the pull-back by $g$ of the hermitian line bundle $\overline{\mathcal{M}}$ on $B$. Then

$$
h_{\overline{\mathcal{L}}}(P)=\frac{1}{m} \widehat{\operatorname{deg}}(\overline{\mathcal{M}})
$$

for all points $P \in V(\overline{\mathbb{Q}})$, and so we immediately obtain one implication in the statement of Theorem 2.

In order to show the reverse implication, we will first show that if $h_{\overline{\mathcal{L}}}$ is bounded then $L$ is torsion in $\operatorname{Pic}(V)$ (this statement is due to L. Szpiro). If $V$ is a smooth projective variety, this statement follows from [La] Chapter 5, Theorem 6.5. If $V$ is normal and projective then by resolution of singularities, there is a morphism $\pi: V^{\prime} \rightarrow V$ with $V^{\prime}$ a smooth projective variety, which is an isomorphism over an open subscheme $U \subset V$ with complement of codimension $\geq 2$. If $h_{\overline{\mathcal{L}}}$ is bounded then the height function $h_{\pi^{*}(\overline{\mathcal{L}})}$ on $V^{\prime}(\overline{\mathbb{Q}})$ is also bounded. Also $\pi^{*}: \operatorname{Pic}(V) \rightarrow \operatorname{Pic}\left(V^{\prime}\right)$ is injective because, since $V$ is normal, $\operatorname{Pic}(V) \rightarrow$ $\operatorname{Pic}(U)$ is injective for $U$ as above. Hence, if $V$ is projective and normal the statement follows. A similar argument, using Chow's lemma, now shows that the statement for $V$ proper and normal follows from the statement for $V$ projective and normal.

Now assume that $h_{\overline{\mathcal{L}}}$ is constant. By the above, it follows that $L$ is torsion, and so by replacing $\overline{\mathcal{L}}$ by a suitable power, we may assume that $L$ is trivial. Hence, there is a rational section $s$ of $\mathcal{L}$ which is a trivialization over some open $U \subset X$ with fibral complement. Denote by $S$ the finite set of places of $K$ consisting of the image of $X-U$ under $g$ together with all the archimedean places. For each $v \in S$, the "norm" of $s$ defines a map

$$
f_{v}=-\log |s|_{v}: X\left(\bar{K}_{v}\right) \rightarrow \mathbb{R}
$$

which is Galois-invariant and continuous for the $v$-adic topology. We will show that each $f_{v}$ is constant. Fix $v \in S$. If $f_{v}$ is not constant, there are Galois invariant $v$-adic open subsets $Z_{v}$ and $Z_{v}^{\prime}$ of $X\left(\bar{K}_{v}\right)$, and $\varepsilon>0$, such that $\mid f_{v}(x)-$ $f_{v}(y) \mid>\varepsilon$ for all $x \in Z_{v}$ and $y \in Z_{v}^{\prime}$. Now by Rumely's density theorem (see $[\mathrm{Ru}]$, p.128), we can find points $P$ and $Q$ in $X\left(\mathcal{O}_{\bar{K}}\right)$, all of whose conjugates lie in $Z_{v}$ and $Z_{v}^{\prime}$ respectively, and such that for all other places $w$ in $S$, all 
conjugates of $P$ and $Q$ lie within a $w$-adic ball of radius $\delta$, for any prescribed $\delta>0$. Since $h_{\overline{\mathcal{L}}}(P)=\sum_{w \in S} h_{w}(P)$ where $h_{w}(P)$ is the average value of $f_{w}$ over the conjugates of $P$, it follows that $h_{\overline{\mathcal{L}}}(P) \neq h_{\overline{\mathcal{L}}}(Q)$ if $\delta$ is small enough, which is a contradiction.

We have now shown that for each place $v \in S$, the function $f_{v}$ is constant, equal to $c_{v} \in \mathbb{R}$, say. Therefore, for each finite place $v \in S$ we can write $f_{v}=-m_{v} \cdot \log \left|\pi_{v}\right|_{v}$ for some $m_{v} \in \mathbb{Q}$ (here $\pi_{v}$ is a uniformizer for $\mathcal{O}_{K_{v}}$ ). Denote by $m$ the l.c.m of the denominators of $m_{v}$. The above identity shows that

$$
m \cdot \operatorname{div}(s)=\left(m m_{v}\right) \cdot X_{v}
$$

where $X_{v}$ denotes the special fiber of $g$ over $v$. Denote by $\mathcal{P}_{v}$ the prime ideal of $\mathcal{O}_{K}$ which corresponds to the finite place $v$. Let us consider the hermitian line bundle $\overline{\mathcal{M}}$ on $B$ which corresponds to the fractional ideal $\mathcal{A}=\prod_{v \in S_{\mathrm{fin}}} \mathcal{P}_{v}^{m m_{v}}$ of $K$ with hermitian metrics given by $\|1\|_{v}=e^{-m c_{v}}$, for $v \in S_{\infty}$. We obtain that there is an isomorphism

$$
\overline{\mathcal{L}}^{\otimes m} \simeq g^{*} \overline{\mathcal{M}}
$$

which is given by the section $s$. Furthermore, if $h_{\overline{\mathcal{L}}}=0$, then equation (1) implies that $\widehat{\operatorname{deg}}(\overline{\mathcal{M}})=0$. This completes the proof of Theorem 2 .

An easy consequence of Theorem 2 is:

Corollary 3. Suppose that for every integer ring $R$ and point $P: \operatorname{Spec}(R) \rightarrow X$ the hermitian line bundle $P^{*} \overline{\mathcal{L}}$ is torsion in the Arakelov class group of $\operatorname{Spec}(R)$. Then $\overline{\mathcal{L}}$ is torsion.

Proof. If $P^{*} \overline{\mathcal{L}}$ is torsion for all points $P$, then $h_{\overline{\mathcal{L}}}$ is identically zero. Therefore, by Theorem 2, a power $\overline{\mathcal{L}}^{\otimes m}$ of $\overline{\mathcal{L}}$ is isomorphic to a pull-back $g^{*} \overline{\mathcal{M}}$ of a hermitian line bundle $\overline{\mathcal{M}}$ on $B$. Now if $P: \operatorname{Spec}(R) \rightarrow X$ is a point of $X, P^{*} \overline{\mathcal{L}}^{\otimes m}$ is isomorphic to the pull-back of $\overline{\mathcal{M}}$ via the finite and flat morphism $\operatorname{Spec}(R) \rightarrow$ $\operatorname{Spec}\left(\mathcal{O}_{K}\right)=B$. Since $P^{*} \overline{\mathcal{L}}$ is torsion in $\widehat{\operatorname{Pic}}(\operatorname{Spec}(R))$, its norm

$$
\operatorname{Norm}_{R / \mathcal{O}_{K}}\left(P^{*} \overline{\mathcal{L}}\right) \simeq \overline{\mathcal{M}}^{\otimes\left[R: \mathcal{O}_{K}\right]}
$$

is torsion in $\widehat{\operatorname{Pic}}(B)$. Hence, $\overline{\mathcal{M}}$ and $\overline{\mathcal{L}}^{\otimes m} \simeq g^{*} \overline{\mathcal{M}}$ are also torsion.

Remark. The above statements and proofs of Theorem 2 and Corollary 3 were suggested by L. Moret-Bailly and communicated to us by the referee. In the first version of the paper, a more complicated proof of a statement equivalent to Theorem 2 was given using the existence theorem of Rumely. The proof given above, which uses the stronger "density theorem" of $[\mathrm{Ru}]$, is a simplification of our original proof. 
Proof of Theorem 1. We will argue by contradiction. Let us consider a hermitian line bundle $\overline{\mathcal{L}}$ which satisfies:

$(A R)$ For every integer ring $R$ and point $P: \operatorname{Spec}(R) \rightarrow X$ the hermitian line bundle $P^{*} \overline{\mathcal{L}}$ is trivial in the Arakelov class group of $\operatorname{Spec}(R)$.

We will show that $\overline{\mathcal{L}}$ is trivial in the Arakelov Picard group $\widehat{\operatorname{Pic}}(X)$. Consider the following statements:

A. If $\overline{\mathcal{L}}$ satisfies $(\mathrm{AR})$, then $\mathcal{L}$ is torsion.

B. If $\overline{\mathcal{L}}$ satisfies $(\mathrm{AR})$ and $\mathcal{L}$ is $N$-torsion for $N$ a prime number, then $L$ is trivial.

C. If $\overline{\mathcal{L}}$ satisfies $(\mathrm{AR})$ and $L$ is trivial, then $\overline{\mathcal{L}}$ is trivial.

The statements $\mathrm{A}, \mathrm{B}, \mathrm{C}$ together imply that if a hermitian line bundle $\overline{\mathcal{L}}$ satisfies (AR) then it is trivial, and this implies the Theorem. Indeed, by A the line bundle $\mathcal{L}$ is torsion. Therefore, for some $n, L^{\otimes n}$ is trivial. The hermitian line bundle $\overline{\mathcal{L}}^{\otimes n}$ also satisfies assumption (AR) and we can apply $\mathrm{C}$ to it; we obtain that $\mathcal{L}^{\otimes n}$ is trivial and therefore $\mathcal{L}$ is $n$-torsion. By a successive application of $\mathrm{A}$ and $\mathrm{B}$ to powers $\overline{\mathcal{L}}^{\otimes a}$ with $a \mid n$ we see that $L$ is trivial. Applying $\mathrm{C}$ again gives the statement.

Proof of $A$. This follows directly from Corollary 3 .

Proof of $B$. Suppose that the line bundle $\mathcal{L}$ is $N$-torsion with $N$ a prime number. Choose an isomorphism $\phi: \mathcal{L}^{\otimes N} \simeq \mathcal{O}_{X}$. This equips the line bundle $L$ with a metric \|\|$\|_{\phi}$ given as follows: if $s$ is a local section of $L$, then $\|s\|_{\phi}=\left|\phi\left(s^{\otimes N}\right)\right|^{1 / N}$. Consider the Kummer fppf exact sequence

$$
1 \longrightarrow H^{0}\left(X, G_{m}\right) / H^{0}\left(X, G_{m}\right)^{N} \longrightarrow H^{1}\left(X, \mu_{N}\right) \longrightarrow H^{1}\left(X, G_{m}\right)[N] \longrightarrow 1
$$

The isomorphism $\phi$ defines a $\mu_{N}$-torsor $t: Y \rightarrow X$ whose class in $H^{1}\left(X, \mu_{N}\right)$ lifts the class of $\mathcal{L}$ in $H^{1}\left(X, G_{m}\right)[N]$. Denote by $W$ the generic fiber of $Y$. We are free to consider $W$ and $V$ as varieties over the field of constants $K$ of $V$. We will show that, under the assumption (AR), the geometric torsor $t_{\bar{K}}: W_{\bar{K}} \rightarrow V_{\bar{K}}$ splits completely (i.e. $W_{\bar{K}}$ has $N$ irreducible components). This implies that the pull-back $L_{\bar{K}}$ of $L$ to $V_{\bar{K}}$ is a trivial line bundle. Then, by Hilbert's theorem $90, L$ is trivial on $V$ and this proves the statement.

Let us base change to $K\left(\zeta_{N}\right)$ in order to include the $N$-th roots of unity in the coefficients. For simplicity, we will use a prime to denote base changes from $K$ to $K\left(\zeta_{N}\right)$ (i.e. we will write $V^{\prime}$ instead of $V \otimes_{K} K\left(\zeta_{N}\right)$ etc). Over $K\left(\zeta_{N}\right)$ there is an isomorphism $\mu_{N} \simeq \mathbb{Z} / N \mathbb{Z}$ and we can identify $t^{\prime}: W^{\prime} \rightarrow V^{\prime}$ with a $\mathbb{Z} / N \mathbb{Z}$-cover.

Denote by $\tau_{a}$ the automorphism of $t_{*}^{\prime}\left(\mathcal{O}_{W^{\prime}}\right)$ which is given by the action of $a \in \mathbb{Z} / N \mathbb{Z}$ on $W^{\prime}$. The base-change $L^{\prime}$ of $L$ on $V^{\prime}$ can be identified with

$$
L^{\prime}=\left\{\lambda \in t_{*}^{\prime}\left(\mathcal{O}_{W^{\prime}}\right) \mid \tau_{a}(\lambda)=\zeta_{N}^{a} \lambda\right\}
$$


The isomorphism $\phi^{\prime}: L^{\prime} \otimes N \simeq \mathcal{O}_{V^{\prime}}$ is given by the ring multiplication in $t_{*}^{\prime}\left(\mathcal{O}_{W^{\prime}}\right)$; there is a corresponding metric \|\|$_{\phi^{\prime}}$ on $L^{\prime}$.

Now let $P$ be an $F$-valued point of the generic fiber $V$ with $F$ a number field over $K\left(\zeta_{N}\right)$. We can write the specialization $\operatorname{Spec}(F) \times_{V} W$ of the cover $t^{\prime}: W^{\prime} \rightarrow V^{\prime}$ at $P$, as $\operatorname{Spec}\left(F_{t}\right)$ with $F_{t}$ an $F$-algebra which is an $N$-dimensional $F$-vector space. Consider the Kummer fppf exact sequence

$$
1 \rightarrow \mathcal{O}_{F}^{*} /\left(\mathcal{O}_{F}^{*}\right)^{N} \rightarrow H^{1}\left(\operatorname{Spec}\left(\mathcal{O}_{F}\right), \mu_{N}\right) \rightarrow H^{1}\left(\operatorname{Spec}\left(\mathcal{O}_{F}\right), G_{m}\right)[N] \rightarrow 1
$$

Since the pull-back $P^{*} \mathcal{L}$ is a trivial line bundle, this exact sequence implies that the Kummer extension $F_{t} / F$ can be generated by extracting the $N$-th root of a unit $u(P) \in \mathcal{O}_{F}^{*}$. As in $(*)$ above, the pull-back $P^{*} \mathcal{L}$ can be viewed as an $R$ submodule of $F_{t}$. In fact, we obtain that the pull-back $P^{*} \mathcal{L}$ is $R \cdot u(P)^{1 / N}$. For $\sigma$ : $F \rightarrow \mathbb{C}$, the length of the generator $u(P)^{1 / N}$ at the corresponding complex point of $\operatorname{Spec}\left(\mathcal{O}_{F}\right)(\mathbb{C})$ with respect to the restriction of the metric \|\|$_{\phi^{\prime}}$ of $L^{\prime}$ given above is equal to $|\sigma(u(P))|^{1 / N}$. Its length with respect to the metric obtained by restricting the original given metric \|\|$_{\mathcal{L}}$ of $\overline{\mathcal{L}}$ is then $c(\sigma(P)) \cdot|\sigma(u(P))|^{1 / N}$ where $c$ is a continuous function on $V^{\prime}(\mathbb{C})$ which is bounded away from 0 and infinity (since both the metrics \|\|$_{\mathcal{L}}$ and \|\|$_{\phi^{\prime}}$ are continuous and $V^{\prime}(\mathbb{C})$ is compact). By our initial assumption, there is an isometry $P^{*} \overline{\mathcal{L}} \simeq \overline{\mathcal{O}}_{\operatorname{Spec}\left(\mathcal{O}_{F}\right)}$; it follows that there is a unit $v(P) \in \mathcal{O}_{F}^{*}$ such that $|\sigma(v(P))| \cdot|\sigma(u(P))|^{1 / N}=1 / c(\sigma(P))$ for all $\sigma: F \subset \mathbb{C}$. By replacing $u(P)$ by $u^{\prime}(P)=v(P)^{N} u(P)$ we see that there is a unit $u^{\prime}(P)$ such that $F_{t}$ is generated by $u^{\prime}(P)^{1 / N}$ and such that $\left|\sigma\left(u^{\prime}(P)\right)\right|$ for all $\sigma: F \subset \mathbb{C}$ is bounded independently of $P$ and $F$. As $F$ contains $\zeta_{N}$, we conclude that for each $F$-valued point $P$ of $V^{\prime}$ the extension $F_{t} / F$ obtained by restricting the torsor $t$ to $P$ is obtained by extracting the $N$-th root of a unit $u^{\prime}(P)$ in $\mathcal{O}_{F}^{*}$ with archimedean valuations which are bounded independently of $P$ and $F$.

Now consider all field extensions $F$ of $K\left(\zeta_{N}\right)$ of degree bounded by a number $d$, say. Given any constant $C>0$, there is only a finite number of units with archimedean valuations bounded by $C$ in all these fields (see for example [Si], Theorem 2.1). Therefore, there is a number field $\tilde{K}$ over $K\left(\zeta_{N}\right)$ which contains all the $N$-th roots of all of these units.

We next observe that, using Noether's normalization theorem, we can find a rational map $\psi: V^{\prime}-\rightarrow \mathbb{P}_{K\left(\zeta_{N}\right)}^{n}$ which is generically finite étale. Suppose that $\psi$ is of degree $d$, and consider the composite finite cover of varieties over $K\left(\zeta_{N}\right)$

$$
W^{\prime} \otimes_{K\left(\zeta_{N}\right)} \tilde{K} \rightarrow W^{\prime} \rightarrow V^{\prime}
$$

If the cover $t_{\bar{K}}: W_{\bar{K}} \rightarrow V_{\bar{K}}$ is not completely split then, since $N$ is prime, the varieties $W^{\prime}$ and $V^{\prime}$ are irreducible. The scheme $W^{\prime} \otimes_{K\left(\zeta_{N}\right)} \tilde{K}$ is also irreducible.

There exists a Zariski open subset $U \subset V^{\prime}$ such that $\psi(U)$ is Zariski open in $\mathbb{P}_{K\left(\zeta_{N}\right)}^{n}$ and $\psi \mid U: U \rightarrow \psi(U)$ is finite étale of degree $d$. By the Hilbert irreducibility theorem (for example [La], Chapter 9; see the formulation in loc. 
cit. $\S 5$, especially Prop. 5.2) there is an infinite set $\mathcal{H}$ of $K\left(\zeta_{N}\right)$-rational points of $\psi(U) \subset \mathbb{P}_{K\left(\zeta_{N}\right)}^{n}$ such that, over these points, the fibers of the composite cover

$$
W^{\prime} \otimes_{K\left(\zeta_{N}\right)} \tilde{K}-\rightarrow \mathbb{P}_{K\left(\zeta_{N}\right)}^{n}
$$

are irreducible. The corresponding points of $V^{\prime}$, i.e the points in $\psi^{-1}(\mathcal{H})$, are all defined over number fields of degree $\leq d$ over $K\left(\zeta_{N}\right)$. Let $F$ be the field of definition of one of these points in $\psi^{-1}(\mathcal{H})$. By the discussion above we have

$$
K\left(\zeta_{N}\right) \subset F \subset F_{t} \subset \tilde{K} F
$$

Therefore, $F_{t} \otimes_{K\left(\zeta_{N}\right)} \tilde{K}$ splits (i.e is not a field). Hence, the fiber of $W^{\prime} \otimes_{K\left(\zeta_{N}\right)} \tilde{K} \rightarrow V^{\prime}$ over this point is not irreducible. This is a contradiction, and so it follows that the cover $t_{\bar{K}}: W_{\bar{K}} \rightarrow V_{\bar{K}}$ is split. By the argument above, this completes the proof of $\mathrm{B}$.

Proof of $C$. For this part, we assume that $L$ is trivial. As in the proof of Theorem 2 , let us take a rational section $s$ of $\mathcal{L}$ which trivializes $\mathcal{L}$ on an open subscheme $U \subset X$ with fibral complement. The section $s$ allows us to identify $\mathcal{L}$ with a sheaf of rational functions on $X$. For every $R$-valued point $P: \operatorname{Spec}(R) \rightarrow X$, the pull-back $P^{*} \overline{\mathcal{L}}$ then corresponds to a metrized fractional ideal $\mathcal{A}_{P}$ of $F=\operatorname{Fr}(R)$. Under our assumption (AR), the metrized ideal $\mathcal{A}_{P}$ is isometric to the trivial metrized ideal $R$; i.e there is an element $f_{P} \in \mathcal{A}_{P}$ such that the homomorphism $R \rightarrow \mathcal{A}_{P}$ given by $1 \mapsto f_{P}$ is an isometry. If $w$ is a place of $F$ then the $w$-adic absolute value of $f_{P}$ is bounded independently of $P$ and $F$.

Suppose that $d>0$ is any number. We conclude that for $P$ running over all points of $V$ defined over number fields $F$ of degree over $K$ bounded by $d$, there is a finite number of possible "normal generators" $f_{P}$ of the ideals $\mathcal{A}_{P}$. Therefore, for each $d>0$ there is a number field $M(d)$ which is generated over $K$ by the set of the "normal generators" $f_{P}$ of $P^{*} \overline{\mathcal{L}}$ for all $P$ defined over $F$ with $[F: K] \leq d$.

Now let $d$ be the generic degree of a generically finite rational map $\psi: V-\rightarrow \mathbb{P}_{K}^{n}$. A similar argument as in the proof of $\mathrm{B}$, using the Hilbert irreducibility theorem applied to the cover

$$
V \otimes_{K} M(d) \rightarrow V-\stackrel{\psi}{\rightarrow} \mathbb{P}_{K}^{n}
$$

shows that $V$ has a point $Q$, defined over a number field $L$ over $K$ with $[L: K] \leq$ $d$ which is linearly disjoint from $M(d)$ (i.e $L \otimes_{K} M(d)$ is a field or equivalently $L \cap M(d)=K)$. Take $f_{Q} \in L$ a normal generator of $Q^{*} \overline{\mathcal{L}}$ as before. By the definition of $M(d), f_{Q}$ belongs to $M(d)$. Using the linear disjointness of $L$ and $M(d)$, we see that $f_{Q} \in K$.

Let us now apply the results and notation used in the proof of Theorem 2. By equation (3), we have that $\overline{\mathcal{L}}^{\otimes m}$ is the pull-back of the hermitian line bundle $\overline{\mathcal{M}}$ corresponding to the metrized ideal $\mathcal{A}$ in $K$. It follows that the line 
bundle $Q^{*} \overline{\mathcal{L}}^{\otimes m}$ on $\operatorname{Spec}\left(\mathcal{O}_{L}\right)$ corresponds to the metrized fractional ideal $\mathcal{A} \mathcal{O}_{L}$. Hence, $\mathcal{A} \mathcal{O}_{L}=f_{Q}^{m} \mathcal{O}_{L}$ as metrized ideals, with $f_{Q} \in K$. We can conclude that $\mathcal{A}=f_{Q}^{m} \mathcal{O}_{K}$ and therefore that the rational numbers $m_{v}$, for all $v \in S_{\text {fin }}$, are actually integers. Also, we obtain that $e^{-m c_{v}}=\left|f_{Q}^{m}\right|_{v}^{-1}$ for all $v \in S_{\infty}$.

Now consider the hermitian line bundle $\mathcal{N}$ on $B$ which corresponds to the metrized ideal of $K$ generated by $f_{Q}$ with metrics given by $\|1\| \|_{v}=\left|f_{Q}\right|_{v}^{-1}$. Using the above discussion, we see that the hermitian line bundle $\overline{\mathcal{L}}$ is isomorphic to $g^{*} \overline{\mathcal{N}}$ and is therefore trivial. This completes the proof of $\mathrm{C}$.

\section{Acknowledgment}

We would like to thank A. J. de Jong, N. Katz, B. Poonen, L. Szpiro, and S. Zhang for useful conversations. We would also like to thank the referee for suggesting a simplification of our original proof and for other helpful comments. The first named author was partially supported by NSF grant no. DMS-9700937. He would like to thank Harvard University and the Université de Bordeaux I for their hospitality while part of this work was carried out. The second named author was partially supported by NSF grant no. DMS-9970378 and by a Sloan Research Fellowship.

\section{References}

[A] A. Agboola, Torsion points on elliptic curves and Galois module structure, Invent. Math. 123 (1996), 105-122.

[Bo] J. B. Bost, Potential theory and Lefschetz theorems for arithmetic surfaces, Ann. Sci. École Norm. Sup. 32 (1999), 241-312.

[La] S. Lang, Fundamentals of Diophantine Geometry, Springer-Verlag (1983).

[Mo] L. Moret-Bailly, Groupes de Picard et problèmes de Skolem I, II, Ann. Sci. École Norm. Sup. 22 (1989), 161-179, 181-194.

[P] G. Pappas, On torsion line bundles and torsion points on abelian varieties, Duke Math. J. 91 (1998), 215-224.

[Ru] R. Rumely, Arithmetic over the ring of all algebraic integers, J. Reine Angew. Math. 368 (1986), 127-133.

[Si] J. Silverman, The theory of height functions, Arithmetic Geometry, Springer-Verlag (1986), 151-166.

Dept. of Mathematics, Harvard University, Boston, MA, 02138.

Permanent address: Dept. of Mathematics, University of California, Santa BarBARA, CA 93106.

E-mail address: agboola@math.ucsb.edu

Dept. of Mathematics, Michigan State University, E. Lansing, Mi 48824.

E-mail address: pappas@math.msu.edu 